\title{
Neue Therapieoption bei Älteren mit Mantelzell-Lymphom
}

Patienten mit einem Mantelzell-Lymphom, die nach einet Rituximab-haltigen Therapie als Erhaltungstherapie weiter den monoklonalen Antikörper Rituximab bekommen, haben einen Vorteil: Ihre krankheitsfreie Zeit ist im Vergleich zur Therapie mit Interferon- $\alpha$ um einige Monate länger. Das geht aus den Ergebnissen einer Studie des Europäischen Mantelzell-Lymphom-Netzwerks hervor.

In die randomisierte Studie wurden 560 Patienten aus acht europäischen Ländern aufgenommen. Sie waren im Median 70 Jahre alt und erhielten als Primärtherapie acht Zyklen R-CHOP (Rituximab, Cyclophosphamid, Doxorubicin, Vincristin und Prednisolon) oder sechs Zyklen R-FC (Rituximab, Fludarabin und Cyclophosphamid). 316 Patienten, deren Lymphom durch diese Therapien ganz oder teilweise zurückgedrängt wur- de, erhielten anschließend eine Rituximab-Erhaltungstherapie (eine Injektion alle zwei Monate) oder eine Erhaltungstherapie mit Interferon- $\alpha$ (eine Injektion pro Woche). Beide Erhaltungstherapien wurden gegeben, bis die Krankheit wieder aktiv wurde.

Die Endauswertung der Studie, für die Daten von 274 Patienten berücksichtigt wurden, zeigte einen deutlichen Vorteil für jene Patienten, die als Erhaltungstherapie Rituximab bekommen hatten: Ihre krankheitsfreie Zeit betrug 75 Monate im Vergleich zu 27 Monaten bei den mit Interferon- $a$ behandelten Patienten.

Besonders bedeutsam zeigte sich dieser Unterschied für jene Patienten, die eingangs mit R-CHOP behandelt worden waren: Nach vier Jahren betrug die Gesamtüberlebensrate bei Patienten nach Rituximab $87 \%$. In der Vergleichsgruppe mit Interferon-a lag die Rate mit $63 \%$ signifikant niedriger. Wurden die Daten aller Patienten berücksichtigt, unterschieden sich die Gesamtüberlebensraten nicht.

„Diese überzeugenden Daten etablieren einen neuen Therapiestandard beim Mantelzell-Lymphom“, wird Martin Dreyling von der Medizinischen Klinik III der Universität München und Koordinator des Europäischen Mantelzell-Lymphom-Netzwerks in einer Pressemitteilung der Klinik zitiert. Obwohl Rituximab gegenwärtig noch nicht für die Erhaltungstherapie beim MantelzellLymphom zugelassen sei, stelle diese im Anschluss an eine R-CHOP Induktionstherapie einen neuen, gut verträglichen Therapiestandard für ältere Patienten mit Mantelzell-Lymphom dar, so das Klinikum. Entsprechend sei diese Therapieempfehlung bereits in die nationalen Leitlinien der DGHO aufgenommen worden.

Peter Leiner

Kluin-Nelemans HC et al. Treatment of Older Patients with Mantle-Cell Lymphoma. N Engl J Med 2012; 367(6):520-31.

\section{Bestrahlung des Abdomens erhöht Diabetesrisiko}

Krebslangzeitüberlebende habe ein erhöhtes Risiko für Insulinresistenz und Diabetes, wenn sie als Kinder wegen eines Wilmstumors oder eines Neuroblastoms eine Bestrahlung des Abdomens erhalten haben. Französische Strahlenexperten und Onkologen prüften des-

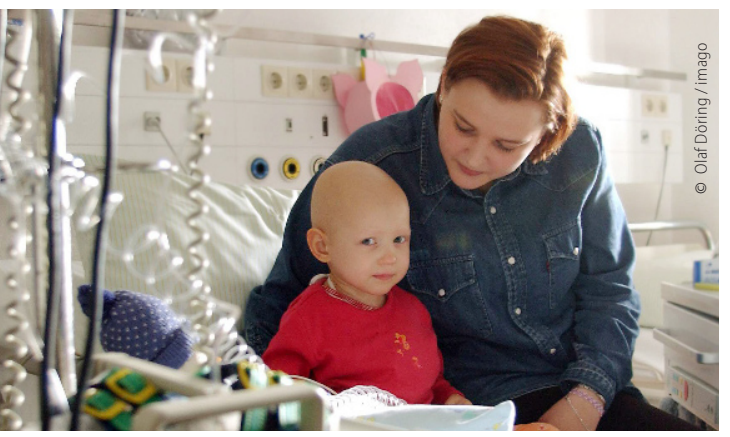

Bei der Bestrahlung des Abdomens von pädiatrischen Krebspatienten sollte versucht werden, den Pankreasschwanz zu schonen. halb die spätere Diabeteswahrscheinlichkeit in Abhängigkeit von dem bestrahlten Abschnitt des Pankreas. Dazu erhielten fast 3.500 Langzeitüberlebende in Frankreich und Großbritannien einen Fragebogen. Alle waren zwischen 1946 und 1985 als Kind wegen eines Tumors bestrahlt worden. Insgesamt 2.520 Fragebögen konnten ausgewertet werden. Die Angaben der Studienteilnehmer zur Bestrahlung und zur Diagnose Diabetes wurden überprüft. 65 der 2.520 Studienteilnehmer hatten einen Diabetes entwickelt.

Die Auswertung der Fragebögen und Befunde ergab, dass das Diabetesrisiko bei der Bestrahlung des Pankreasschwanzes mit einer Dosis von 29 Gy ein Plateau erreichte, bei höheren Strahlendosen also nicht weiter zunahm. Bei Bestrahlung der übrigen Pankreasregionen trat kein signifikanter Effekt auf.
Wurden die Studienteilnehmer als Kinder mit mindestens 10 Gy im Bereich des Pankreasschwanzes, wo die Insulin produzierenden $\beta$-Zellen sitzen, bestrahlt, so war das Risiko, später an Diabetes zu erkranken, im Vergleich zu den nicht bestrahlten Teilnehmern unabhängig von BMI und Geschlecht um das 11,5-Fache erhöht. Bei einer Dosis von 1 Gy betrug das geschätzte relative Risiko 1,61 .

Kinder unter zwei Jahren waren stärker durch die Strahlentherapie gefährdet als ältere Kinder: Das relative Risiko, später an Diabetes zu erkranken, war bei ihnen bei einer Strahlendosis von 1 Gy mit 2,1 versus 1,4 signifikant höher.

Die Autoren empfehlen aufgrund der Studienergebnisse, bei Patienten, die bereits als Kind an Krebs erkrankt waren, verstärkt auf Zeichen eines beginnenden Diabetes zu achten. In Studien sei Diabetes frühestens 20 Jahre nach Behandlung aufgetreten.

Peter Leiner

Vathaire F et al. Radiation dose to the pancreas and risk of diabetes mellitus in childhood cancer survivors: a retrospective cohort study. Lancet Oncol. 2012 Aug 22. [Epub ahead of print] 